\title{
Rigorous electromagnetic analysis of a microcylindrical axilens with long focal depth and high transverse resolution
}

\author{
Bi-Zhen Dong, Juan Liu, Ben-Yuan Gu, and Guo-Zhen Yang \\ Institute of Physics, Academia Sinica, P.O. Box 603, Beijing 100080, China \\ Jian Wang \\ Department of Physics, The University of Hong Kong, Pokfulam Road, Hong Kong, China
}

Received July 17, 2000; accepted November 29, 2000; revised manuscript received January 2, 2001

\begin{abstract}
We first present nonparaxial designs for a microcylindrical axilens with different long focal depths and rigorously analyze electromagnetic field distributions of the axilens using integral equations and the boundaryelement method. Numerical results show that the designed axilenses indeed have the special feature of attaining a long focal depth while keeping high transverse resolution for numerical apertures of 2.4, 2.0, and 1.0. The ratio between the extended focal depth of the designed axilens and the focal depth of the conventional focal lens is 1.41 , the corresponding maximal extended focal depth of the axilens can reach $28 \mu \mathrm{m}$, and the spot size of the focal beam is $\sim 10 \mu \mathrm{m}$ over the focal range. (C) 2001 Optical Society of America
\end{abstract}

OCIS codes: $050.0050,050.1970,220.3620$.

\section{INTRODUCTION}

As is well known, conventional optical focal lenses cannot produce a beam with long focal depth and narrow lateral width simultaneously. In recent years, various axilenses and axicons have been suggested as diffractive optical elements with a special function that modulates the incident light wave to produce a constant axial intensity distribution and narrow lateral width within a given long focal range. ${ }^{1-8}$ This kind of element can be widely used for high-precision alignment in some branches of optical and mechanical engineering, in optical disk readout, in magneto-optical storage devices, and so on. However, all approaches and analyses for determining the phase function of the axilens or axicons have been in the paraxial case and based on scalar-diffraction analysis methods. To our knowledge, the design problem of an axilens in the nonparaxial case based on rigorous electromagnetic analysis has not been discussed in the literature up to now. This motivates us to construct a phase function in the nonparaxial case for the axilens with long focal depth and high transverse resolution on the basis of vector diffraction theory.

With the development of microfabrication technology, diffractive microlenses can be fabricated practically with use of modern microlithography. Diffractive microlenses, refractive microlenses, and microlens arrays with submicrometer-sized structure in integrated micro-optoelectro-mechanical systems (MOEMs) have been extensively investigated. The microaxilens with a long focal depth and high transverse resolution in MOEMs is also important, and we are motivated to study and analyze its performance.

In this paper we present, as far as we know for the first time, a rigorous electromagnetic analysis of a microax- ilens with long focal depth and high transverse resolution. We develop the method in the paraxial case presented by Davidson et al. ${ }^{1}$ to construct a phase function in the nonparaxial case for designing a microcylindrical axilens with a long focal length. We numerically study and analyze the optical performance of the axilens on the basis of vector diffractive theory and a rigorous solution of the electromagnetic equations. ${ }^{9-13}$ The numerical simulations are implemented with the use of the boundaryelement method (BEM). ${ }^{14-17}$

This paper consists of four sections. In Section 2 the basic formulas and method are described for the boundary integral equations. In Section 3 we present nonparaxial designs for a microcylindrical axilens with a long focal depth and analyze the rigorous numerical results. A brief summary is given in Section 4 .

\section{INTEGRAL EQUATIONS}

The two-dimensional scattering problem in a cylindrical axilens system is shown in Fig. 1, in which the boundary $\Gamma$ with a curved surface structure divides the twodimensional space into two semi-infinite regions $S_{1}$ and $S_{2}$, and each of the regions $S_{i}(i=1,2)$ is filled by a homogeneous material with refractive index $n_{i}(i=1,2)$. The unit vector $\hat{n}$ is normal to the boundary $\Gamma$ and is oriented to region $S_{1}$. A plane light wave in space is normally incident on the boundary $\Gamma$ on region $S_{1}$ and then travels into region $S_{2}$ along the $y$-axis direction, i.e., the axis of the focal axilens. To determine the transmitted electric field distribution in region $S_{2}$, we apply Green's formula, the radiation condition, and Maxwell's equations to obtain the boundary integral equations ${ }^{9}$ : 


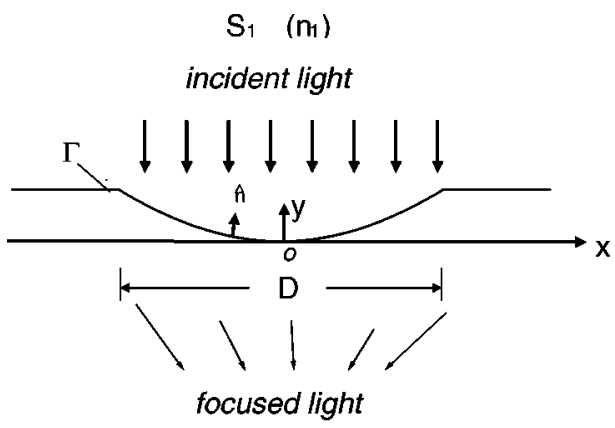

$S_{2}\left(n_{2}\right)$

Fig. 1. Schematic diagram of a two-dimensional scattering problem in a cylindrical system.

$$
\begin{aligned}
-E_{1}\left(\mathbf{r}_{1}\right)+ & \int_{\Gamma}\left[E_{1}\left(\mathbf{r}_{\Gamma}^{\prime}\right) \hat{n} \cdot \nabla G_{1}\left(\mathbf{r}_{1}, \mathbf{r}_{\Gamma}^{\prime}\right)\right. \\
& \left.-G_{1}\left(\mathbf{r}_{1}, \mathbf{r}_{\Gamma}^{\prime}\right) \hat{n} \cdot \nabla E_{1}\left(\mathbf{r}_{\Gamma}^{\prime}\right)\right] \mathrm{d} l^{\prime}=-E^{\mathrm{inc}}\left(\mathbf{r}_{1}\right), \\
E_{2}\left(\mathbf{r}_{2}\right)+ & \int_{\Gamma}\left[E_{2}\left(\mathbf{r}_{\Gamma}^{\prime}\right) \hat{n} \cdot \nabla G_{2}\left(\mathbf{r}_{2}, \mathbf{r}_{\Gamma}^{\prime}\right)\right. \\
& \left.-G_{2}\left(\mathbf{r}_{2}, \mathbf{r}_{\Gamma}^{\prime}\right) \hat{n} \cdot \nabla E_{2}\left(\mathbf{r}_{\Gamma}^{\prime}\right)\right] \mathrm{d} l^{\prime}=0 .
\end{aligned}
$$

The boundary conditions on $\Gamma$ are given by $E_{1}=E_{2}$ and $\hat{n} \cdot \nabla E_{1}=\hat{n} \cdot \nabla E_{2}$.

To solve these equations, two unknown quantities, the local fields $E_{i}$ and the corresponding normal derivatives $\nabla E_{i}$ on the boundary, must first be evaluated. We assume that $\mathbf{r}_{1}$ and $\mathbf{r}_{2}$ approach a point $\mathbf{r}_{\Gamma}$ on $\Gamma$; therefore Eqs. (1) and (2) can be expressed as follows ${ }^{10-12}$ :

$$
\begin{gathered}
\left(\frac{\theta_{\Gamma}}{2 \pi}-1\right) E_{1}\left(\mathbf{r}_{\Gamma}\right)+\int_{\Gamma}\left[E_{1}\left(\mathbf{r}_{\Gamma}^{\prime}\right) \hat{n} \cdot \nabla G_{1}\left(\mathbf{r}_{\Gamma}, \mathbf{r}_{\Gamma}^{\prime}\right)\right. \\
\left.-G_{1}\left(\mathbf{r}_{\Gamma}, \mathbf{r}_{\Gamma}^{\prime}\right) \hat{n} \cdot \nabla E_{1}\left(\mathbf{r}_{\Gamma}^{\prime}\right)\right] \mathrm{d} l^{\prime}=-E^{\operatorname{inc}}\left(\mathbf{r}_{\Gamma}\right), \\
\left(\frac{\theta_{\Gamma}}{2 \pi}\right) E_{2}\left(\mathbf{r}_{\Gamma}\right)+\int_{\Gamma}\left[E_{2}\left(\mathbf{r}_{\Gamma}^{\prime}\right) \hat{n} \cdot \nabla G_{2}\left(\mathbf{r}_{\Gamma}, \mathbf{r}_{\Gamma}^{\prime}\right)\right. \\
\left.-G_{2}\left(\mathbf{r}_{\Gamma}, \mathbf{r}_{\Gamma}^{\prime}\right) \cdot \nabla E_{2}\left(\mathbf{r}_{\Gamma}^{\prime}\right)\right] \mathrm{d} l^{\prime}=0,
\end{gathered}
$$

where all the notation in Eqs. (1)-(4) is defined in Table 1 and the integral indicates Cauchy's principal values of integration. Here the Green function

$$
G_{i}\left(\mathbf{r}_{i}, \mathbf{r}_{\Gamma}^{\prime}\right)=(-j / 4) H_{0}^{(2)}\left(k_{i}\left|\mathbf{r}_{i}-\mathbf{r}_{\Gamma}^{\prime}\right|\right) \quad(i=1,2)
$$

is used, where $H_{0}^{(2)}$ is the zero-order Hankel function of the second kind and $k_{i}=n_{i} k_{0}(i=1,2)$, where $k_{0}$ is the free-space wave number, $k_{0}=2 \pi / \lambda$, and $\lambda$ is the wavelength of the incident light in free space.

To obtain the solutions of $E_{i}$ and $\nabla E_{i}$, we first employ the BEM to solve Eqs. (3) and (4). The numerical approach of the BEM generally consists of the following steps $^{10,15-17}$ :

(a) The boundary $\Gamma$ is divided into $m$ quadratic elements, and each quadratic element includes three nodes. The optical field and its derivative over each element are assumed to vary according to the interpolation functions $N_{i}(i=1,2,3)$,

$$
\begin{aligned}
& N_{1}=\xi(\xi-1) / 2, \\
& N_{2}=(1-\xi)(1+\xi), \\
& N_{3}=\xi(1+\xi) / 2,
\end{aligned}
$$

where $\xi=x, y$.

(b) Equations (3) and (4) can then be cast into the form of a set of linear equations by expanding $E\left(\mathbf{r}_{\Gamma}\right)$ and $\nabla E\left(\mathbf{r}_{\Gamma}\right)$ over the quadratic elements as

$$
\begin{gathered}
E\left(\mathbf{r}_{\Gamma}\right)=\left\{\begin{array}{l}
N_{1} \\
N_{2} \\
N_{3}
\end{array}\right\}\left\{E^{\mathrm{I}}\left(\mathbf{r}_{\Gamma}\right), E^{\mathrm{II}}\left(\mathbf{r}_{\Gamma}\right), E^{\mathrm{III}}\left(\mathbf{r}_{\Gamma}\right)\right\}, \\
\nabla E\left(\mathbf{r}_{\Gamma}\right)=\left\{\begin{array}{l}
N_{1} \\
N_{2} \\
N_{3}
\end{array}\right\}\left\{\nabla E^{\mathrm{I}}\left(\mathbf{r}_{\Gamma}\right), \nabla E^{\mathrm{II}}\left(\mathbf{r}_{\Gamma}\right), \nabla E^{\mathrm{III}}\left(\mathbf{r}_{\Gamma}\right)\right\},
\end{gathered}
$$

where $E^{\mathrm{I}(\mathrm{II}, \mathrm{III})}\left(\mathbf{r}_{\Gamma}\right)$ and $\nabla E^{\mathrm{I}(\mathrm{II}, \mathrm{III})}\left(\mathbf{r}_{\Gamma}\right)$ are the electric field values and their normal derivatives at the three nodes of each element.

(c) The integral over each element is carried out by using, in general, a numerical quadrature scheme. By solving these linear equations, we can obtain the local field $E_{i}$ and the normal derivative of the local field $\nabla E_{i}$.

(d) The total electric field at any point on region $S_{2}$ can thus be numerically calculated from Eq. (2).

In conventional design, one employs a rectangular window as the aperture of the axilens, and the profile of the incident plane wave is abruptly truncated by this window. This causes the incident beam to diffract into different directions such that the incident light cannot be better focused by the axilens. Therefore the diffraction efficiency of the axilens is rapidly reduced, and considerable energy of the incident light is lost. To alleviate these losses, a window function with smoothly varying edge profile is introduced in our design. The window function is characterized by a parameter $s$ and is given by ${ }^{10}$

Table 1. Definition of Notation

\begin{tabular}{cl}
\hline Notation & \multicolumn{1}{c}{ Definition } \\
\hline$\Gamma$ & Boundary between region $S_{1}$ and $S_{2}$ \\
$\mathbf{r}_{1(2)}$ & Position vector in region $S_{1(2)}$ \\
$\mathbf{r}_{\Gamma}\left(\mathbf{r}_{\Gamma}^{\prime}\right)$ & Position vector on the boundary $\Gamma$ \\
$d l^{\prime}$ & Integral line element along the boundary $\Gamma$ \\
$\hat{n}$ & Normal unit vector \\
$E_{1(2)}\left(\mathbf{r}_{1(2)}\right)$ & Total field in region $S_{1(2)}$ \\
$E_{1(2)}\left(\mathbf{r}_{\Gamma}^{\prime}\right)$ & Local field at the boundary $\Gamma$ \\
$\nabla E_{1(2)}\left(\mathbf{r}_{\Gamma}^{\prime}\right)$ & from region $S_{1(2)}$ approaching $\Gamma$ \\
& Normal derivative of the local field \\
$E^{\text {inc }}\left(\mathbf{r}_{1}\right)$ & at the boundary $\Gamma$ from region $S_{1(2)}$ \\
$G_{1(2)}\left(\mathbf{r}_{1(2)}, \mathbf{r}_{\Gamma}^{\prime}\right)$ & approaching $\Gamma$ \\
$\nabla G_{1(2)}\left(\mathbf{r}_{1(2)}, \mathbf{r}_{\Gamma}^{\prime}\right)$ & Green function in region $S_{1(2)}$ \\
$\theta_{\Gamma}$ & Gradient Green function in region $S_{1(2)}$ \\
\hline
\end{tabular}




$$
w(x)= \begin{cases}1 & 0 \leqslant|x| \leqslant \frac{D}{2} \\
\exp \left[-10\left(|x|-\frac{D}{2}\right)^{2}\right] & \begin{array}{l}
D \\
\frac{2}{2} \leqslant|x| \leqslant \frac{D}{2}+s, \\
0
\end{array} \\
\frac{D}{2}+s<|x|<\infty\end{cases}
$$

where $D$ denotes the size of axilens aperture and $s$ $=1 \mu \mathrm{m}$ is taken in our numerical calculations.

\section{NUMERICAL SIMULATIONS}

\section{A. Design of a Microcylindrical Axilens with Long Focal Depth}

The phase function of the conventional focal cylindrical lens can be approximately expressed as a quadratic term in the paraxial case, ${ }^{1,18}$

$$
\phi(x)=\frac{2 \pi}{\lambda} \frac{x^{2}}{2 f},
$$

where $f$ is the focal length of the lens and $x$ is the coordinate on the lens plane. If, instead of a constant focal length $f$, the axilens were composed of zones each of which possessed different focal length parameters in its phase function expression, the entire depth-of-focus of element would be enlarged. If we choose these zones to be concentric rings with infinitesimal width, the axilens phase function can be rewritten as

$$
\phi(x)=\frac{2 \pi}{\lambda} \frac{x^{2}}{2 f(x)},
$$

where $f(x)$ is a continuous function instead of a constant; its form was suggested by Davidson et al. ${ }^{1}$ as follows:

$$
f(x)=f_{0}+\delta y \frac{x^{2}}{R^{2}},
$$

where $2 R=D$ and $f_{0}$ stand for the size of the aperture and the starting focal length of the axilens, respectively, and $\delta y$ denotes a predesigned focal depth over which the intensity along the $y$ axis is required to be a constant distribution.

In the nonparaxial case, the phase function of the focal lens is written as ${ }^{10,18}$

$$
\phi(x)=\left(\frac{n_{2}}{n_{1}-n_{2}}\right)\left(f^{2}+x^{2}\right)^{1 / 2} .
$$

Substituting Eq. (11) into Eq. (12), we can obtain the phase function of the axilens in the nonparaxial case as follows:

$$
\phi(x)=\left(\frac{n_{2}}{n_{1}-n_{2}}\right)\left[\left(f_{0}+\delta y \frac{x^{2}}{R^{2}}\right)^{2}+x^{2}\right]^{1 / 2} .
$$

The parameters $f_{0}$ and $R$ in Eq. (13) can be chosen according to a specific application, and $\delta y$ takes a preset value. The extended focal depth of the axilens can be increased when the preset value of the focal depth $\delta y$ is increased.
When the incident plane wave goes through the axilens and is modulated by this phase function of the axilens, then the incident field, $E^{\text {inc }}$ in Eqs. (1) and (3) involves the phase function of Eq. (13) and is expressed as

$$
E^{\text {inc }}=w(x) \exp [\phi(x)] .
$$

Therefore, when the predesigned value of the focal depth $\delta y$ is changed, the distribution of the total field is also changed.

\section{B. Numerical Results}

Assume that the size of the aperture of the axilens is $D$ $=30 \mu \mathrm{m}$ and that the starting focal length of the axilens, $f_{0}$ is taken as $60 \mu \mathrm{m}$. In our calculations the refractive indices $n_{1}$ and $n_{2}$ are taken as 1.5 for glass material and 1.0 for air, respectively. The preset focal depth $\delta y$ is taken to be 30 and $60 \mu \mathrm{m}$. We employ these parameters to demonstrate the expanded focal depth of the focused field and the lateral resolution of the focused beam over $S_{2}$ region.

We now calculate the intensity distributions of the total electric field, including both the axial and the lateral directions according to Eqs. (1)-(4) with the use of the BEM to demonstrate the optical performance of the axilens with different parameters. A plane wave with wavelength $\lambda=1 \mu \mathrm{m}$ in free space is normally incident on the microcylindrical axilens along a negative direction of the $y$ axis, as shown in Fig. 1 .

The characteristics around the focal region for the designed axilens are shown in Figs. 2 and 3. Figure 2 displays the axial intensity distribution along the $y$ axis, i.e., $I(x=0, y)$, for different preset values of $\delta y$; curves a and b correspond to $\delta y=30$ and $60 \mu \mathrm{m}$, respectively. For a comparison, we also calculated the axial intensity distribution for a conventional focal lens of $\delta y=0$ that has the same aperture $D$ and a focal length $f_{0}$, as shown by curve c. By comparing curves a and b with curve $c$ we can easily observe that the shapes of curves $a$ and $b$ become wider than that of curve c. This means that the focal depth of the axilens has indeed increased with an increase in the value of $\delta y$, but the extended focal depth cannot reach the preset value. If we consider an intensity greater than $80 \%$ of maximal intensity as the average value of the axial intensity, then the corresponding extent along the $y$ axis can be reasonably regarded as the extended focal depth of the axilens, which is drawn by the dashed lines in Fig. 2. The extended focal depths of the axilens are 18.5 and $22.0 \mu \mathrm{m}$ for curves a and b, respectively. However, the focal depth of the conventional lens is only $15.2 \mu \mathrm{m}$. The ratio between the extended focal depth of the designed axilens and the conventional focal depth is 1.22 (1.45) for curve a (b). The focal planes corresponding to maximal intensity peak for curves $a, b$, and c in Fig. 2 are located at $y=-61.5,-67.6$, and $55.5 \mu \mathrm{m}$, respectively.

To observe the lateral intensity distributions on the planes for different distances from the microaxilens surface, we calculated the lateral intensity of the axilens of $\delta y=60 \mu \mathrm{m}, I(x, y=$ const. $)$, at three different distances from the axilens. The obtained results are shown in Fig. 3 for different values of $y$ : The solid curve corresponds to $y=-58 \mu \mathrm{m}$, the dashed curve to $y=-67 \mu \mathrm{m}$, and the 
dotted curve to $y=-81 \mu \mathrm{m}$. It is evident that the axilens exhibits the good characteristic that the total intensity distribution of the electric fields is always concentrated on the central lobe, which is of a size close to the diffraction-limited spot size $2.44 \lambda(F / D) \approx 4.8 \mu \mathrm{m}$.

To present a global view of both the axial intensity distribution and the lateral resolution, we prefer to display the field patterns in a 256 gray-level representation in Fig. 4: (a) for $\delta y=30 \mu \mathrm{m}$, (b) for $\delta y=60 \mu \mathrm{m}$, and (c) for $\delta y=0 \mu \mathrm{m}$. The bright (dark) regions indicate the area of the high (low) field intensity. From Figs. 4(a) and 4(b) we can globally see the total electric field intensity condensed around the $y$ axis over the focal range. These figures verify that the designed axilens has the special feature of long focal depth with high transverse resolution.

When the parameters in the design are changed, the important feature of the axilens remains unchanged. For instance, we designed an axilens with the aperture of $D=25 \mu \mathrm{m}$, with all the other parameters retained as above. Figure 5 shows the axial intensity distributions for different values of $\delta y$ : Curves $\mathrm{a}$ and $\mathrm{b}$ are for $\delta y$ $=30$ and $60 \mu \mathrm{m}$, respectively. For comparison, the focal depth for the conventional focal lens $(\delta y=0)$ is also presented, and the corresponding axial intensity distribu-

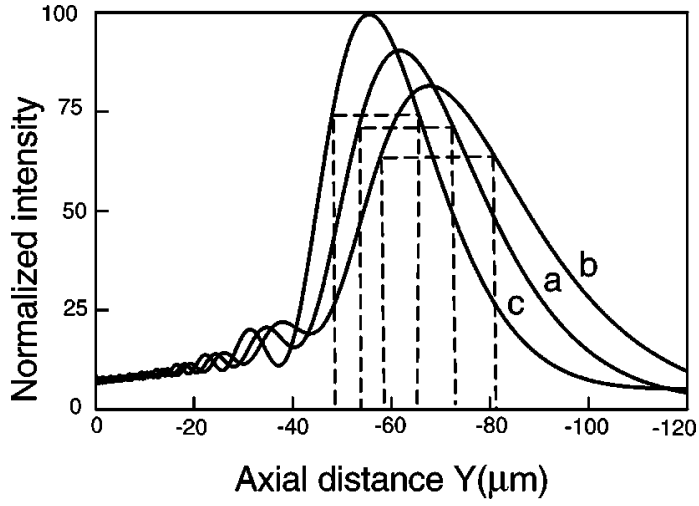

Fig. 2. Diffracted field intensity distribution as a function of the axial distance around the focal region for the axilenses with different designed focal depths, where the size of the aperture of the axilens is $D=30 \mu \mathrm{m}$. Curves a and b are for axilenses with preset focal depths $\delta y=30$ and $60 \mu \mathrm{m}$, respectively; curve $\mathrm{c}$ is for the conventional focal lens, $\delta y=0$.

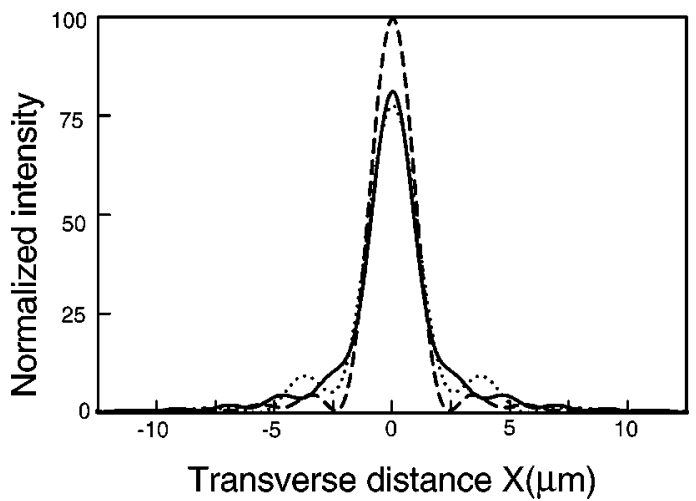

Fig. 3. Diffracted intensity distribution on three planes of different distances from the axilens surface: dashed curve, for $y$ $=-58 \mu \mathrm{m}$; solid curve, for $y=-67 \mu \mathrm{m}$; dotted curve, for $y$ $=-81 \mu \mathrm{m}$. (a)

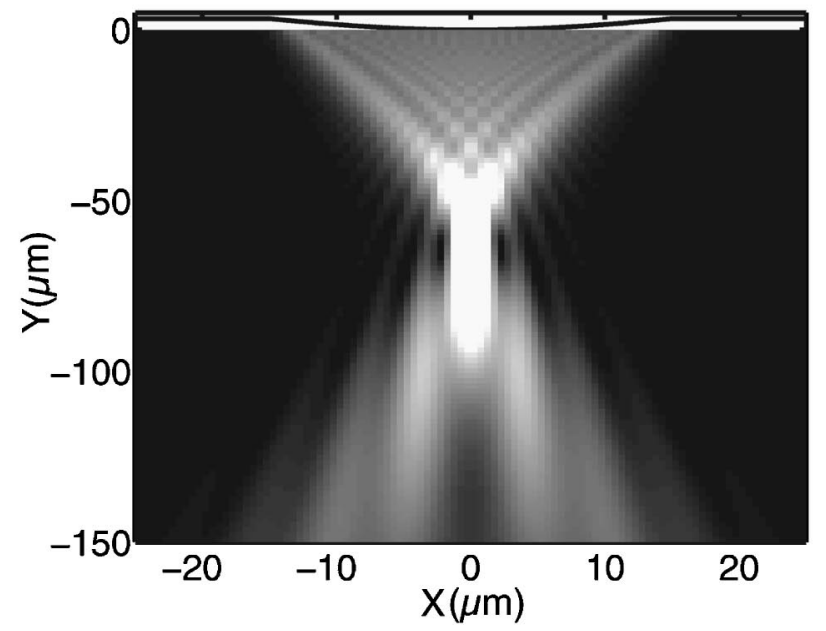

(b)

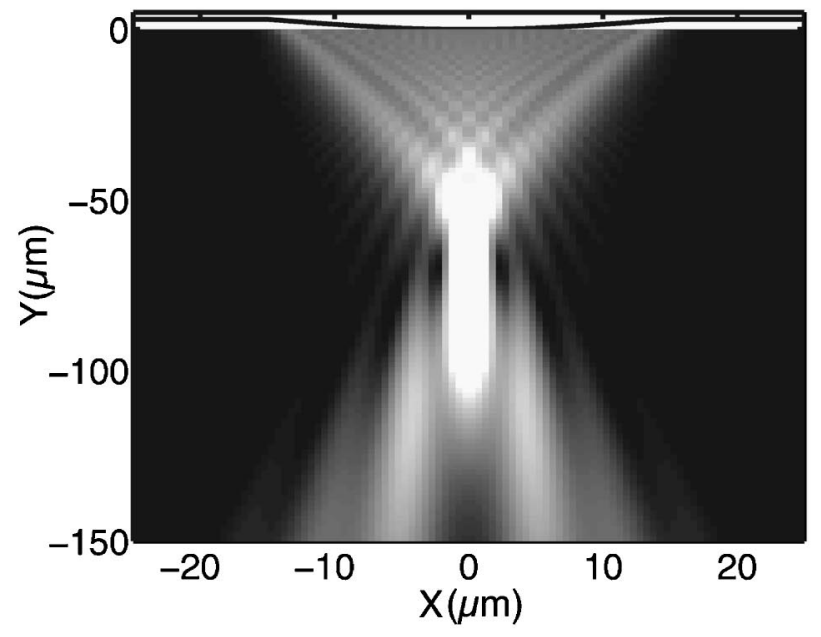

(c)

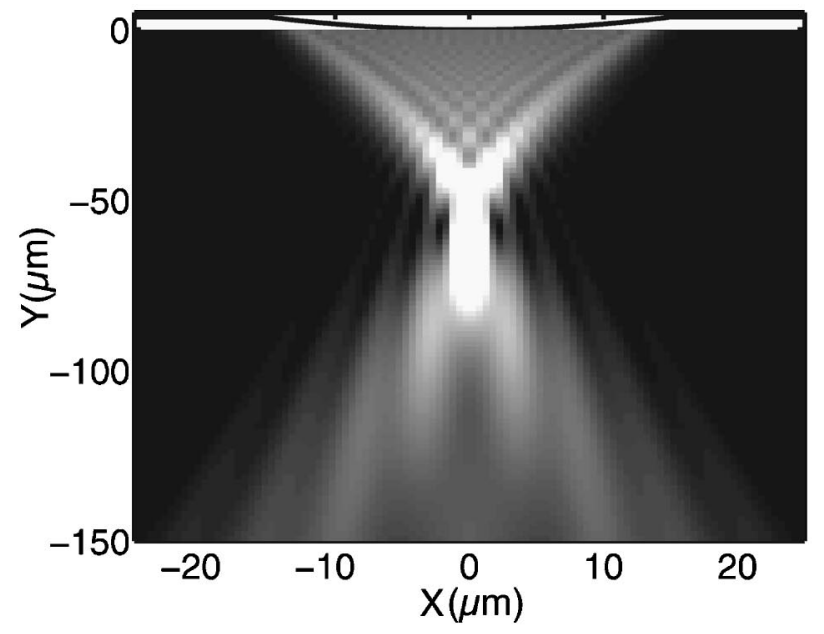

Fig. 4. Intensity distribution of the electric field plotted in a 256 gray-level representation for axilenses with present focal depths (a) $\delta y=30 \mu \mathrm{m}$ and (b) $\delta y=60 \mu \mathrm{m}$, and for (c) the conventional lens, $\delta y=0 \mu \mathrm{m}$. The bright (dark) region indicates the high (low) field intensity areas. 


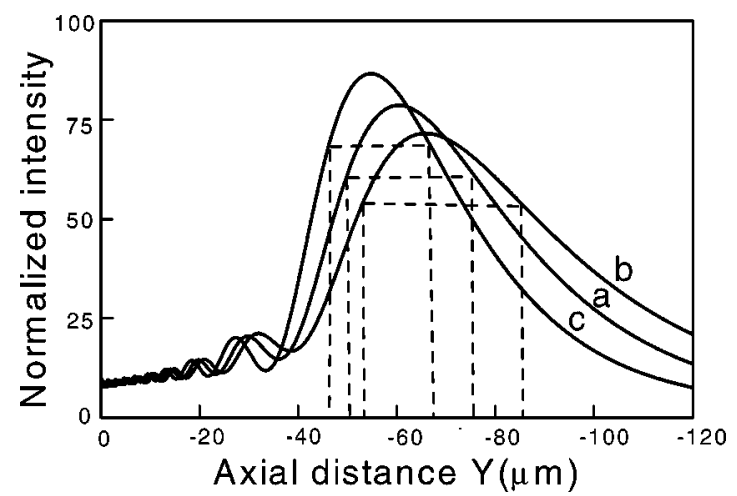

Fig. 5. Axial intensity distribution around the focal range for axilenses with aperture $D=25 \mu \mathrm{m}$ and with different preset focal depth parameters. Curves $a$ and $b$ are for preset focal depths $\delta y=30$ and $60 \mu \mathrm{m}$, respectively; curve $\mathrm{c}$ is for the conventional focal lens, $\delta y=0$.

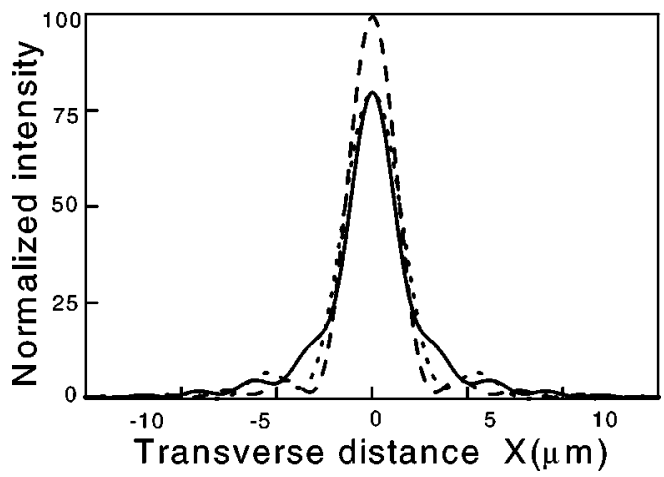

Fig. 6. Lateral intensity distribution on three planes of different distances from the axilens surface: dashed curve, $y$ $=-54 \mu \mathrm{m} ;$ solid curve, $y=-65 \mu \mathrm{m}$; dotted curve, $y$ $=-82 \mu \mathrm{m}$.

tions is plotted as curve c in Fig. 5. The extended focal depths are $24.0 \mu \mathrm{m}$ for curve a and $28.3 \mu \mathrm{m}$ for curve b. The focal depth of the conventional lens is only $20.1 \mu \mathrm{m}$. The ratio between the extended focal depth of the designed axilens and the conventional focal depth is 1.19 (1.41) for curve a (b). When curve a (b) in Fig. 5 is compared with curve a (b) in Fig. 2, it is evident that the extended focal depths in Fig. 5 are larger than those in Fig. 2 , at the expense of a reduction in intensity along the $y$ axis. These results are expected because of the reduction in the size of the aperture. Focal planes with maximal intensity peaks appear at $y=-60.3,-65.8$, and -54.7 $\mu \mathrm{m}$ for curves $\mathrm{a}, \mathrm{b}$, and c, respectively in Fig. 5 . Figure 6 describes the lateral intensity distribution for the axilens of $\delta y=60 \mu \mathrm{m}$ at three different planes within the focal range. Solid, dashed, and dotted curves correspond to the lateral intensity distributions at the planes $y$ $=-54,-65$, and $-82 \mu \mathrm{m}$, respectively. Thus it can be seen clearly that the total intensity distribution of the electric fields is always condensed on the central lobe, on which the spot size is $10.54 \mu \mathrm{m}$, larger than the diffraction-limited spot size of $5.86 \mu \mathrm{m}$.

We present another 256 gray-level representation in Fig. 7: Figs. 7(a), 7(b), and 7(c) correspond to $\delta y=30$, 60 , and $0 \mu \mathrm{m}$, respectively. From Fig. 7 it is globally seen that the focal extent of the focused beam is expanded and is longer than that in Fig. 4 but that the spot size is wider than that in Fig. 4.

The numerical apertures (NA's) in Figs. 2 and 5 are taken as 2.0 and 2.4, respectively. We also investigate

(a)

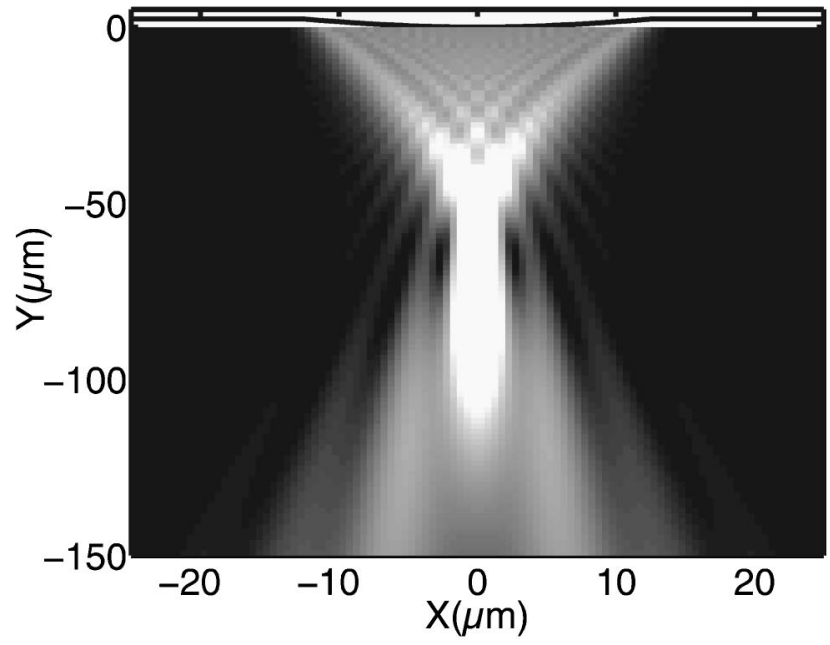

(b)

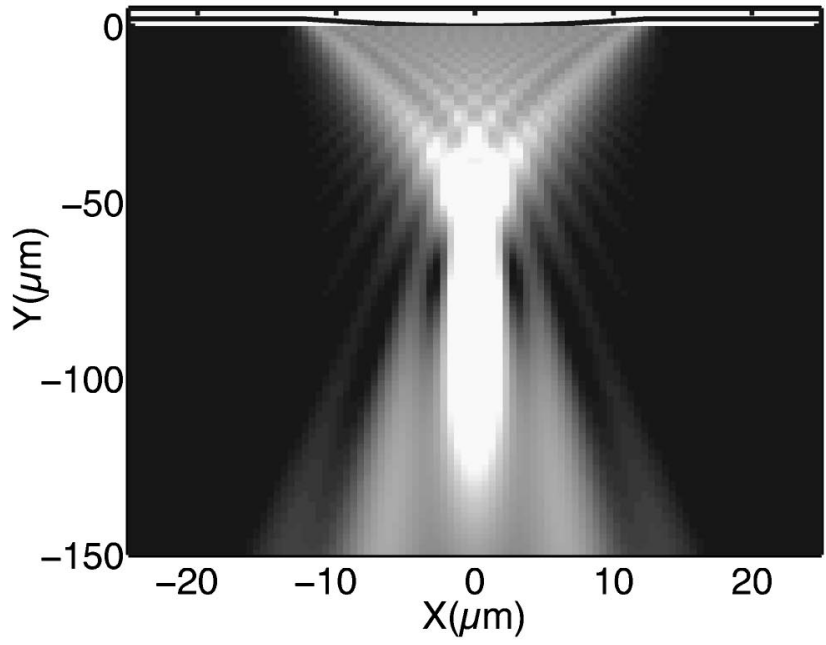

(c)

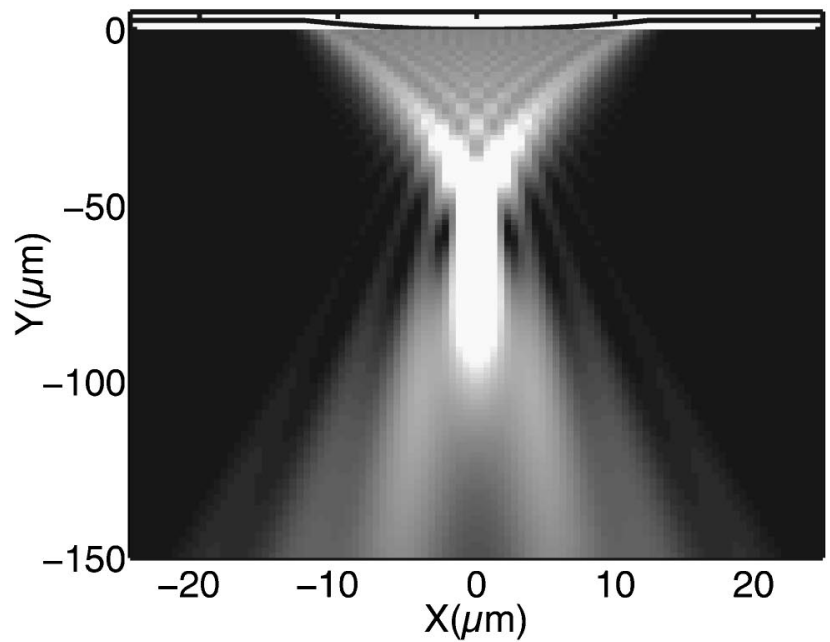

Fig. 7. Same as Fig. 4, except that the aperture of the axilens is $25 \mu \mathrm{m}$. 


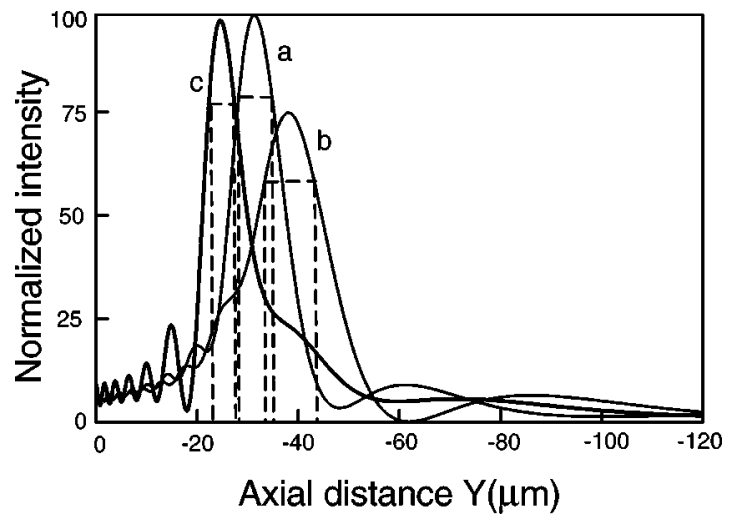

Fig. 8. Axial intensity distribution around the focal region for an axilens of NA $=1.0$. Curves $a$ and $b$ are for preset focal depths $\delta y=30$ and $60 \mu \mathrm{m}$, respectively; curve $\mathrm{c}$ is for the conventional focal lens, $\delta y=0 \mu \mathrm{m}$.

the optical performance of the microaxilens for the case of high $f$-number, for instance, $\mathrm{NA}=1.0$. We reduce $f_{0}$ to $30 \mu \mathrm{m}$; the other parameters are the same as in Fig. 2 . Figure 8 shows the axial intensity distribution along the $y$ axis, i.e., $I(x=0, y)$, for different preset values of $\delta y$ $=30,60$, and $0 \mu \mathrm{m}$ for curves $\mathrm{a}, \mathrm{b}$, and $\mathrm{c}$, respectively. The extended focal depths of the axilens are 6.6 and 9.1 $\mu \mathrm{m}$ for curves a and b, respectively, and the focal depth of the conventional lens is $5.2 \mu \mathrm{m}$, corresponding to curve c. The ratio between the extended focal depth of the axilens and the conventional focal depth is 1.27 (1.75) for curve a (b). It is evident that the extended focal depth for NA $=1.0$ in Fig. 8 is shorter than that for $\mathrm{NA}=2.0$ in Fig. 2 even though the preset focal depth is the same. These results verify that the microaxilens still has good optical performance with long focal depth and narrow transverse resolution even though NA is reduced to 1.0. However, if $\mathrm{NA}$ is reduced to 0.5 , the optical performance of the axilens deteriorates.

\section{SUMMARY}

In this paper we have presented nonparaxial designs of the microaxilens and implemented a rigorous electric magnetic analysis for the microaxilens with the use of integral equations and the boundary-element method. The results of numerical simulations have shown that the designed axilens has the special feature of long focal depth and high transverse resolution simultaneously for NA $=2.4,2.0$, and 1.0. The maximal extended focal depth can reach $28 \mu \mathrm{m}$, and the corresponding ratio between the extended focal depth and the conventional focal depth is 1.41. The extended focal depth of the axilens is increased when the predesignated focal depth parameter is increased. However, the extended depth of focus cannot reach the preset value.

It is expected that the microaxilens with long focal depth and high transverse resolution may be very useful for practical applications in micro-opto-electromechanical systems.

\section{ACKNOWLEDGMENTS}

This work was supported by the Major State Basic Research Development Program "Integrated Micro-Optical-
Electro-Mechanical Systems" under contract G1999033104 in China. B.-Z. Dong is grateful for the hospitality of the Department of Physics, The University of Hong Kong, China.

Corresponding author Bi-Zhen Dong can be reached by fax, 86-10-8264-9531, or e-mail, bzdong@aphy.iphy.ac.cn.

\section{REFERENCES}

1. N. Davidson, A. A. Friesem, and E. Hasman, "Holographic axilens: high resolution and long focal depth," Opt. Lett. 16, 523-525 (1991).

2. J. Sochacki, S. Bará, Z. Jaroszewicz, and A. Kołodziejczyk, "Phase retardation of the uniform-intensity axilens," Opt. Lett. 17, 7-9 (1992).

3. J. Sochacki, A. Kołodziejczyk, Z. Jaroszewicz, and S. Bará, "Nonparaxial design of generalized axicons," Appl. Opt. 31, 5326-5330 (1992)

4. L. F. Staroński, J. Sochacki, Z. Jaroszewicz, and A. Kołodziejczyk, "Lateral distribution and flow of energy in uniformintensity axicons," J. Opt. Soc. Am. A 9, 2091-2094 (1992).

5. J. Sochacki, Z. Jaroszewicz, L. R. Staroński, and A. Kołodziejczyk, "Annular-aperture logarithmic axicon," J. Opt. Soc. Am. A 10, 1765-1768 (1993).

6. Z. Jaroszewicz, J. Sochacki, A. Kołodziejczyk, and L. R. Staroński, "Apodized annular-aperture logarithmic axicon smoothness and uniformity of intensity distributions," Opt. Lett. 18, 1893-1895 (1993).

7. B.-Z. Dong, G.-Z. Yang, and B.-Y. Gu, "Iterative optimization approach for designing an axicon with long focal depth and high transverse resolution," J. Opt. Soc. Am. A 13, 97103 (1996).

8. R. Kant, "Superresolution and increase depth of focus: an inverse problem of vector diffraction," J. Mod. Opt. 47, 905916 (2000).

9. K. Yashiro and S. Ohkawa, "Boundary element method for electromagnetic field problems," IEEE Trans. Antennas Propag. AP-33, 383-389 (1985).

10. K. Hirayama, E. N. Glytsis, T. K. Gaylord, and D. W. Wilson, "Rigorous electromagnetic analysis of diffractive cylindrical lenses,” J. Opt. Soc. Am. A 13, 2219-2231 (1996).

11. J. M. Bendickson, E. N. Glytsis, and T. K. Gaylord, "Scalar integral diffraction methods: unification, accuracy, and comparison with a rigorous boundary-element method with application to diffractive cylindrical lenses," J. Opt. Soc. Am. A 15, 1822-1837 (1998).

12. K. Hirayama, K. Igarashi, Y. Hayashi, E. N. Glytsis, and T. K. Gaylord, "Finite-substrate-thickness cylindrical diffractive lenses: exact and approximate boundary-element methods," J. Opt. Soc. Am. A 16, 1294-1302 (1999).

13. E. N. Glytsis, M. E. Harrigan, T. K. Gaylord, and K. Hirayama, "Effects of fabrication errors on the performance of cylindrical diffractive lenses: rigorous boundaryelement method and scalar approximation," Appl. Opt. 37, 6591-6602 (1998)

14. E. N. Glytsis, M. E. Harrigan, K. Hirayama, and T. K. Gaylord, "Collimating cylindrical diffractive lenses: rigorous electromagnetic analysis and scalar approximation," Appl. Opt. 37, 34-43 (1998).

15. M. Koshiba, Optical Waveguide Theory by the Finite Element Method (KTK Scientific, Tokyo, 1992), pp.43-47.

16. R. Petit, ed., Electromagnetic Theory of Gratings (SpringerVerlag, Berlin, 1980), Chap. 6.

17. S. Kagami and I. Fukai, "Application of boundary-element method to electro-magnetic field problems," IEEE Trans. Microwave Theory Tech. MTT-32, 455-461 (1984).

18. D. A. Buralli, G. M. Morris, and J. R. Rogers, "Optical performance of holographic kinoforms,” Appl. Opt. 28, 976-983 (1989). 